\title{
Caracterización de la composición y fluctuaciones en la densidad y diversidad de los ensambles planctónicos en cuatro humedales de Tarapacá.
}

\author{
Esteban Quinán $^{\mathrm{a}^{* *}}$, Juan Pablo Oyandel ${ }^{\mathrm{b}}$, Karina Díaz ${ }^{\mathrm{b}}$, Carolina Vera ${ }^{\mathrm{a}}$ \\ ${ }^{a}$ Centro de Estudios de Humedales-Centro de Estudios del Desarrollo. \\ * Autor para correspondencia, email: esteban.quinan@gmail.com \\ b Laboratorio de Limnología, Departamento de Ciencias Ecológicas, Universidad de Chile.
}

\section{Resumen}

La limnología de los humedales interiores en Tarapacá presenta ciclos productivos altamente dependientes de las lluvias estivales y las condiciones fisicoquímicas del medio. Entre 2013 y 2015 se estudiaron ensambles planctónicos de dos lagunas de evaporación y dos quebradas, durante dos temporadas productivas y dos vegetativas, junto con esto se analizaron las condiciones fisicoquímicas asociadas. Los resultados mostraron que el fitoplancton en general estuvo dominado por diatomeas y sólo en una quebrada por algas verdes, mientras que el zooplancton presentó composiciones muy distintas entre las lagunas y entre las quebradas, con importantes variaciones temporales. En lagunas, las densidades altas de zooplancton se asociaron positivamente con sólidos disueltos totales y su diversidad se asoció inversamente con la temperatura. Por otra parte, la diversidad de fitoplancton mantuvo una relación inversa con la riqueza de zooplancton.

Palabras clave: Lagunas, quebradas, zooplancton, fitoplancton, diversidad, parámetros de agua.

\begin{abstract}
The limnology of the interior wetlands in Tarapacá presents productive cycles highly dependent on the summer rains and the physicochemical conditions of the environment. Between 2013 and 2015 planktonic assemblies of two evaporation lagoons and two ravines were studied during two productive and two vegetative seasons, together with the associated physicochemical conditions. The results show that phytoplankton in general has been dominated by diatoms and only in a green algae ravine, while zooplankton presented very different compositions between the lagoons and between the ravines, with important temporal variations. In ponds, zooplankton high densities were positively associated with total dissolved solids and their diversity was inversely associated with temperature. On the other hand, phytoplankton diversity maintained an inverse relationship with the richness of zooplankton.
\end{abstract}


Sustainability, Agri, Food and Environmental Research, 4(4), 2016: 27-44

ISSN: 0719-3726

Keywords: lagoons, streams, zooplankton, phytoplankton, diversity, water parameters.

\section{INTRODUCCION}

El altiplano de Tarapacá, a una elevación promedio de 3700 metros sobre el nivel del mar y como parte de la provincia de la Puna (Morrone, 2002), presenta un clima de marcada estacionalidad. Durante los meses del invierno austral, la humedad atmosférica es particularmente baja, pero durante los meses del verano se caracteriza por manifestar un régimen pluvial de carácter torrencial, concentrado en cortos períodos de tiempo (Aceituno 1997), con efectos directos sobre los ecosistemas a menor altitud, producto de los escurrimientos superficiales y la recarga de los acuíferos (Peña et al., 1989). En este contexto climático de estepa altoandina (Gajardo 1994), paisaje semi-arido y biológicamente uniforme, se destaca la presencia de humedales asociados a salares y depósitos salinos (Niemeyer \& Cereceda, 1984; De los Ríos-Escalante, 2010; De los Ríos-Escalante et al., 2013). Éstos están formados en cuencas cerradas donde la evaporación potencial siempre es superior a la recarga freática, por lluvias o por derretimiento de nieves (Keller \& Soto 1998), como espacios que albergan ecosistemas complejos y variados. Por otra parte a menor altitud pero presentes en todo el rango altitudinal, las quebradas son accidentes geográficos determinantes en el balance hídrico de las cuencas, ya que es en éstas donde ocurren las infiltraciones y afloramientos regulares que dan origen a bofedales (sensu Risacher et al., 1999), formaciones de vegetación siempreverde hidrófila y en cojinetes compactos. Ambos tipos de ecosistemas, de evaporación y escurrimiento, están sometidos cíclicamente a drásticas variaciones en sus niveles y parámetros, producto de la constante alternancia entre periodos húmedos y periodos secos. Además son ambientes de alto valor tanto por su oferta de hábitat para la biodiversidad, como por los servicios que proveen a la población humana.

En Chile se sabe, en general, que los ensambles de crustáceos zooplanctónicos en aguas interiores se caracterizan por una baja riqueza de especies y por la presencia, tanto de especies cosmopolitas como de endémicas (De Los Ríos-Escalante, 2010). La mayor parte de las descripciones sobre fitoplancton se han concentrado históricamente en ecosistemas del centro y sur de Chile, con particular interés en la flora diatomológica (Rivera, 2006).También se han escrito importantes inventarios de los sistemas límnicos del altiplano (Araya \& Zúñiga, 1985; De Los Ríos-Escalante, 2010), pero en el caso de los ecosistemas de quebrada y sus condiciones del agua asociadas, la literatura disponible es escasa.

Con el objetivo de describir la composición de los ensambles planctónicos en humedales y sus relaciones con el medio abiótico en Tarapacá, se realizaron muestreos estandarizados en dos lagunas altoandinas y dos quebradas, durante estaciones lluviosas y secas entre los años 2013 y 2015. 
Sustainability, Agri, Food and Environmental Research, 4(4), 2016: 27-44

ISSN: 0719-3726

\section{MATERIALES Y MÉTODOS}

Sitios de estudio: se estudiaron simultáneamente cuatro humedales andinos, dos lagunas altiplánicas endorreicas de alta evapotranspiración y dos quebradas. La laguna salar del Huasco (3.800 msnm), con un centro de cuenca de $60 \mathrm{~km}$ de largo por $30 \mathrm{~km}$ de ancho, se caracteriza por ser uno de los salares menos intervenidos que quedan en la macro zona norte de Chile. La Laguna Lagunillas (4020 msnm), distante unos $58 \mathrm{~km}$ al norte de Huasco, es un sistema intervenido desde 1994 para la extracción de agua con fines industriales y desde el año 2006 es recargada artificialmente con agua subterránea para mantención del espejo de agua y el bofedal adyacente. En cuanto a las quebradas, el sitio de Iquiuca-Parca $(2550 \mathrm{msnm})$, es un ecosistema antropizado pero casi despoblado, ubicado al fondo de la sub-subcuenca de Quipisca, entre laderas abruptas de orientación oesteeste, sobre el piso ecológico de precordillera, con vegetación de tipo arbórea, arbustiva y herbácea. Finalmente, en quebrada Copaquire (3550 msnm), de orientación oeste-este, el humedal estudiado se ubica en una zona de transición entre altiplano y precordillera, con solo una habitante que hace ocupación con ganadería de subsistencia.

Parámetros fisicoquímicos: se midieron ocho parámetros fisicoquímicos in situ en estaciones fijas dentro de cada humedal, en los meses de diciembre 2013, mayo 2014, diciembre 2014 y mayo 2015. En las lagunas se tomaron muestras en tres puntos sobre vertientes y tres puntos en el espejo de agua, mientras que en cada una de las quebradas se establecieron dos puntos de muestreo (tabla 1 y figura 1). Para comprobar la diferenciación primaria entre ambientes considerados salinos y de agua dulce en los humedales de laguna, se compararon los valores de salinidad obtenidos en las vertientes y en el espejo de agua.

Tabla 1. Coordenadas de las estaciones de muestreo en cuatro humedales de la región de Tarapacá. Laguna del Huasco (HL) y sus vertientes (HV), laguna Lagunillas (LL) y sus vertientes (LV), quebrada de Copaquire (C) y quebrada de Iquiuca-Parca (IP).

\begin{tabular}{cccccc}
\hline Estación & UTM X & UTM Y & Estación & UTM X & UTM Y \\
\hline HL1 & 511692 & 7756081 & LL3 & 516115 & 7795732 \\
HL2 & 511803 & 7757136 & LV1 & 515924 & 7796395 \\
HL3 & 512277 & 7758148 & LV2 & 515879 & 7796433 \\
HV1 & 516280 & 7751473 & LV3 & 515739 & 7796313 \\
HV2 & 511532 & 7757208 & HC1 & 511940 & 7685702 \\
HV3 & 512720 & 7758828 & HC2 & 511687 & 7685694 \\
LL1 & 515938 & 7795824 & IP1 & 477528 & 7785881 \\
LL2 & 516095 & 7795781 & IP2 & 476935 & 7785718 \\
\hline \hline
\end{tabular}




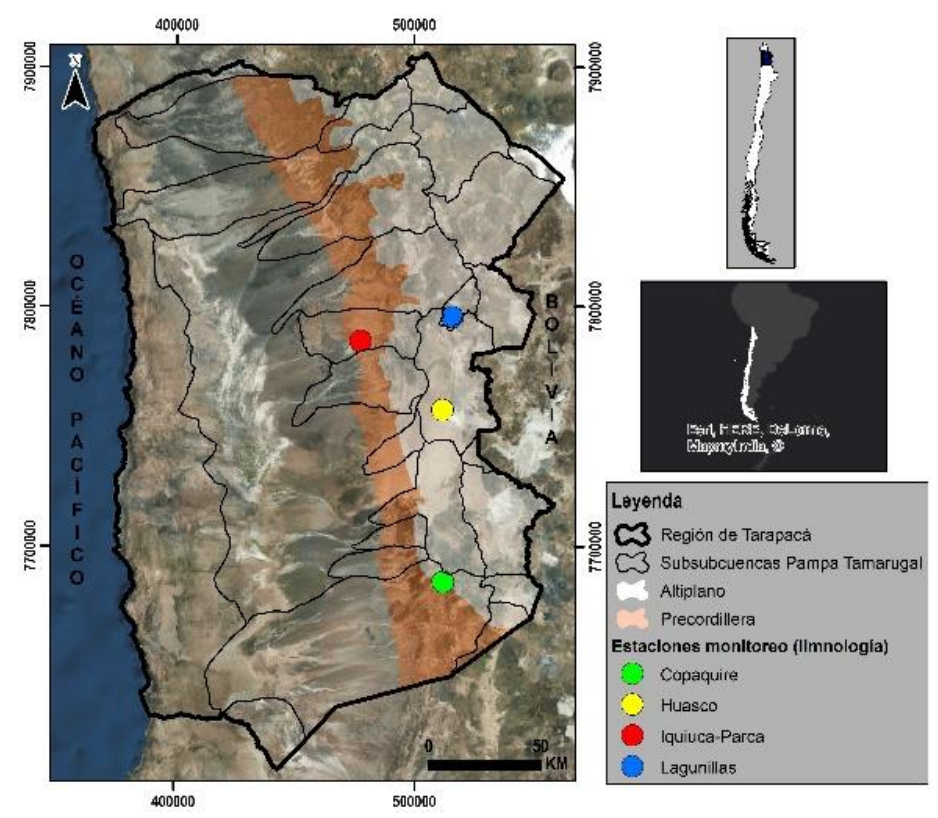

Figura 1. Imagen satelital de la región de Tarapacá, donde se indican los cuatro humedales en estudio, distribuidos entre los pisos ecológicos de precordillera y altiplano.

Componentes bióticos: en cada estación, para estudiar fitoplancton se tomaron muestras de agua de $1 \mathrm{~L}$ fijadas en lugol 90\% y analizadas mediante concentración en cámaras de sedimentación (Utermöhl, 1958) siguiendo a Elosegi \& Sabater (2009). La identificación taxonómica a nivel de géneros se basó en las descripciones de Cox (1996), Parra \& Bicudo (1995, 1996), Parra et al. (1982, 1983), Bicudo \& Menezes (2005), Sant'Anna et al. (2006). La abundancia de organismos fue calculada según la fórmula de Arocena \& Conde (1999), N: CA/aSV donde, C: número de organismos contados; A: área del fondo de la cámara; a: área del campo contado; $\mathrm{S}: \mathrm{n}^{\circ}$ de campos contados; V: volumen de la cámara. Para estudiar el zooplancton, en cada estación se colectaron 3 réplicas de 15 litros tamizados $(200 \mu \mathrm{m})$ y se fijaron en alcohol 70\%. Las muestras se analizaron con lupa estereoscópica Wild M3 y cámara de conteo Bogorov. La identificación taxonómica se realizó usando las descripciones de Araya \& Zuñiga (1985), Villalobos (2006), Williamson \& Reid (2001), Wallace \& Snell (2001), De los Rios \& Salgado (2012) y Chapman \& Burns (1976).

Para las muestras de zoo y fitoplancton en cada monitoreo, se observaron las densidades totales de individuos o células, que fueron promediadas para representar la densidad en cada humedal por temporada. Los ensambles se caracterizaron usando índices de riqueza taxonómica $\left(\mathrm{S}^{\prime}\right)$, Shannon-Wiener $\left(\mathrm{H}^{\prime}\right)$, Pielou $\left(\mathrm{J}^{\prime}\right)$ y relación de distancia de Jaccard. La caracterización fisicoquímica de cada humedal se hizo mediante la comparación de medias con U-Mann Whitney y análisis de componentes principales. Para observar correlaciones entre las variables bióticas de cada ensamble y los parámetros de agua asociados se realizó un 
Sustainability, Agri, Food and Environmental Research, 4(4), 2016: 27-44

ISSN: 0719-3726

análisis multivariado. Los análisis estadísticos se realizaron usando los softwares Biodiversity Pro V.2 (MacAleece, 1997), R (R Core Team, 2015) y XLStat (Addinsoft, 2004).

\section{Resultados}

Parámetros de agua observados en los cuatro humedales: en Huasco la salinidad media en las vertientes $(\mathrm{X}=0,43 \mathrm{ppt} ; \mathrm{SD}=0,38$ ) (Mann-Whitney unilateral; $\mathrm{U}=118 ; \mathrm{P}=0,004)$ fue menor y más estable que en el espejo de la laguna $(X=16,78$ ppt; $S D=28,62)$. En Lagunillas, también se hallaron diferencias entre la salinidad observada en las vertientes $(X=0,20 ; S D=0,14)$ y la laguna $(X=0,39 ; S D=0,36)$, pero menos significativas (Mann-Whitney; $\mathrm{U}=107 ; \mathrm{P}=0,042$ ). Por otra parte, al comparar los niveles de salinidad observados en las quebradas de Iquiuca-Parca $(X=0,62 \mathrm{ppt} ; \mathrm{SD}=0,05)$ y Copaquire $(\mathrm{X}=0.60 ; \mathrm{SD}=0,05)$ no se halló diferencia estadística (Mann-Whitney; $\mathrm{U}=13 ; \mathrm{P}=0,154)$. Si bien, en el cuerpo de agua de laguna Lagunillas la salinidad presentó variaciones similares a las observadas en el salar del Huasco, ésta se mantuvo en rangos comparables con lo observado en sistemas de agua dulce (vertientes y quebradas). Mientras que, en laguna del Huasco alcanzó valores hasta dos órdenes de magnitud más altos que los otros tres humedales muestreados (tabla 2 y figura 2).

El análisis integrado de las mediciones in situ en los cuatro humedales, durante las cuatro temporadas climáticas, revela que las vertientes de ambos salares y el cuerpo de la laguna Lagunillas son sistemas altamente comparables desde el punto de vista de las condiciones fisicoquímicas en sus aguas, no solo desde el punto de vista de su salinidad. El espejo de Huasco se diferenció notoriamente de laguna Lagunillas, en tanto que los sistemas de quebrada presentaron la mayor variabilidad, reflejando un rango de condiciones más amplio en las lagunas (figura 3).

Tabla 2. Salinidad media (ppt) en ambientes de laguna y vertientes de los humedales de Huasco y Lagunillas. L es laguna y $\mathrm{V}$ es vertiente.

\begin{tabular}{lcccc} 
& Huasco L & Huasco V & Lagunillas L & Lagunillas V \\
\hline Diciembre 2013 & 39,74 & 0,27 & 0,77 & 0,14 \\
Mayo 2014 & 3,44 & 0,36 & 0,29 & 0,14 \\
Diciembre 2014 & 2,99 & 0,35 & 0,14 & 0,37 \\
Mayo 2015 & 20,96 & 0,72 & 0,36 & 0,14 \\
\hline \hline
\end{tabular}




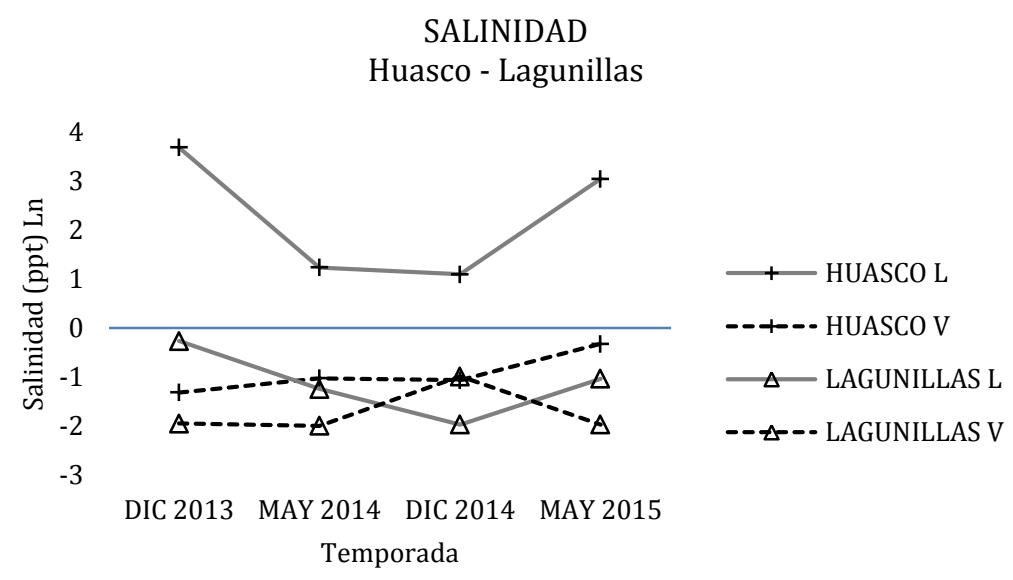

Figura 2. Niveles de salinidad observados en los ambientes de vertiente y laguna, para los humedales de Huasco y Lagunillas. Se presenta el logaritmo natural de los valores medios observados en cada temporada.

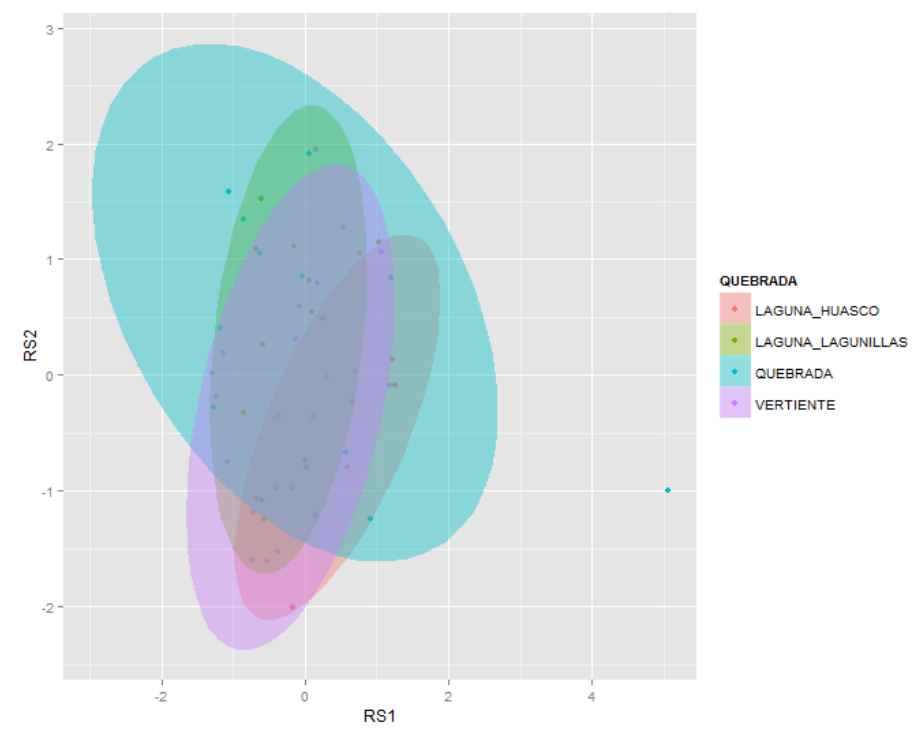

Figura 3. Análisis de componentes principales (ACP) sobre los parámetros físico-químicos medidos in situ en lagunas, vertientes y quebradas de Tarapacá. Las elipses reúnen el 75\% de la variabilidad.

Composición y estructura de los ensambles planctónicos: en la porción fitoplanctónica de los cuatro humedales se identificaron en total 51 taxa, pertenecientes a seis clases, siendo las lagunas los ambientes más diversos.

Los humedales de Huasco y Lagunillas fueron los ambientes más diversos $\left(H^{\prime}=0,83\right.$ y 0,81 respectivamente). En términos taxonómicos, los ensambles de Huasco y Lagunillas son muy similares entre sí $(57,14 \%)$ y bien diferenciados de los ensambles en las quebradas Copaquire e Iquiuca-Parca $(47,05 \%$ similitud 
entre sí) (figura 4). Aunque ambas lagunas estuvieron dominadas por las diatomeas Nitzschia y Diatoma (Bacillariophyceae > 99\% DR en ambas), las fracciones menos densas de algas verdes (Chlorophyceae) y verdeazules (Cyanophyceae) contribuyeron con una mayor diversidad ( $\mathrm{H}^{\prime}>0,8$ y $\mathrm{J}^{\prime}=0,54$ en ambas). El ensamble de quebrada Copaquire también estuvo altamente dominado por diatomeas (Nitzschia 98,04\% DR, J'=0.18), pero con muy pocas especies acompañantes $\left(\mathrm{H}^{\prime}=0,25 ; \mathrm{J}^{\prime}=0,18\right)$. Quebrada Iquiuca-Parca albergó una estructura comunitaria muy distinta de los otros tres humedales, dominada básicamente por Chlorophyceae (DR=75,57\%) y donde las diatomeas fueron taxa secundarios (figura 5). Charophyceae (Klebsormidium sp) solo se identificaron en las quebradas, durante mayo y diciembre 2014; Cryptophyceae se detectaron exclusivamente en las vertientes de Huasco y Lagunillas durante diciembre 2013; y Euglenophyceae solo en Lagunillas durante mayo 2015. Choococcus solo se detectó en la quebrada de Iquiuca-Parca en diciembre 2013.
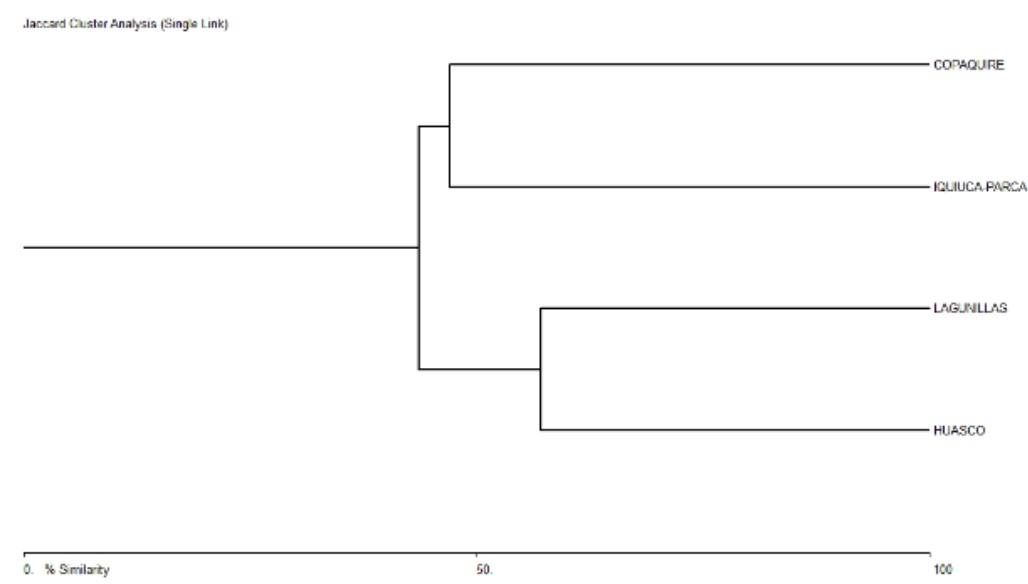

Figura 4. Análisis de clusters sobre la composición del fitoplancton en cuatro humedales de Tarapacá, observada de diciembre 2013 a mayo 2015. Relación de distancia de Jaccard.

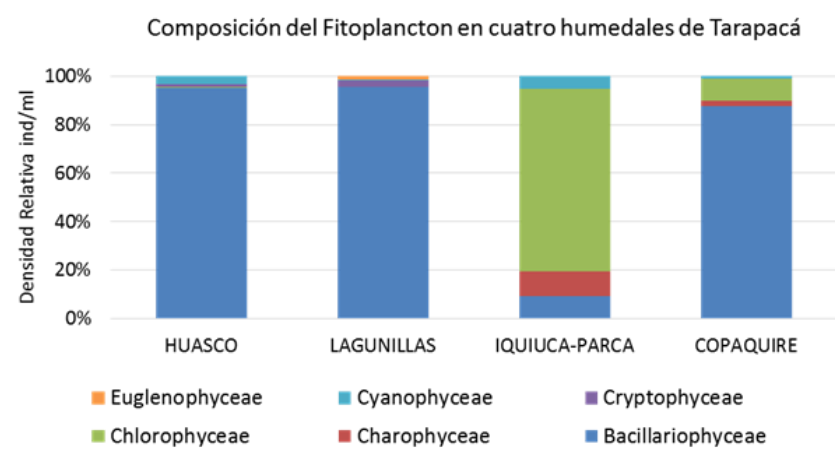

Figura 5. Composición taxonómica en el fitoplancton de cuatro humedales de Tarapacá observada de diciembre 2013 a mayo 2015, como densidad relativa (DR). 
En la porción zooplanctónica se identificaron en total 18 taxa, pertenecientes a 7 familias de crustáceos y tres familias de rotíferos, donde al menos la mitad de la riqueza identificada es compartida por los cuatro humedales, con Lagunillas y Copaquire altamente similares (> 72\%) y Huasco como el humedal más diferente (figura 6).

Lagunillas fue el humedal más diverso y de mayor distribución $\left(\mathrm{H}^{\prime}=0,75, \mathrm{~J}^{\prime}=0,72\right)$, con Rotifera como taxa dominante $(\mathrm{DR}=44,23 \%)$, seguido por copépodos y cladóceros en densidades comparables. En Huasco y las quebradas los ensambles estuvieron dominados por Cladocera (DR >79\% en los tres) (figura 7). El humedal de Copaquire resultó ser el menos diverso $\left(\mathrm{H}^{\prime}=0,25\right)$, dominado particularmente por cladóceros Chydoridae en mayo 2014. Se destaca la alta densidad de Daphnia sp registrada en el humedal de Huasco durante mayo 2015.

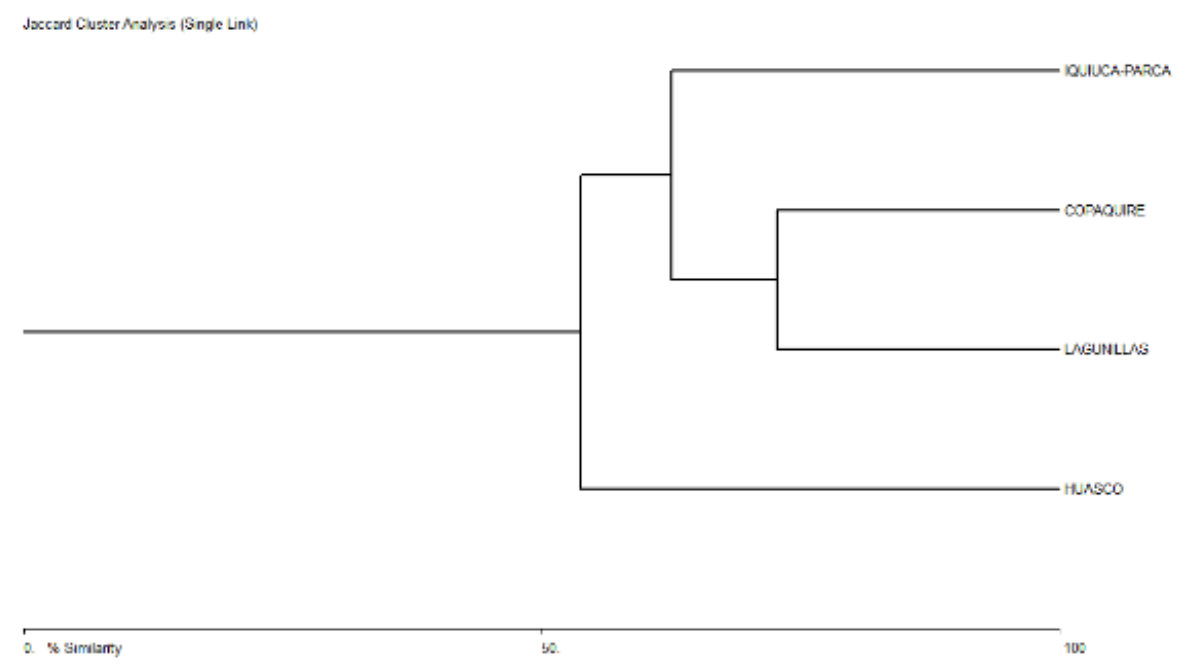

Figura 6. Análisis de clusters sobre la composición del zooplancton en cuatro humedales de Tarapacá, observada de diciembre 2013 a mayo 2015. Relación de distancia de Jaccard.

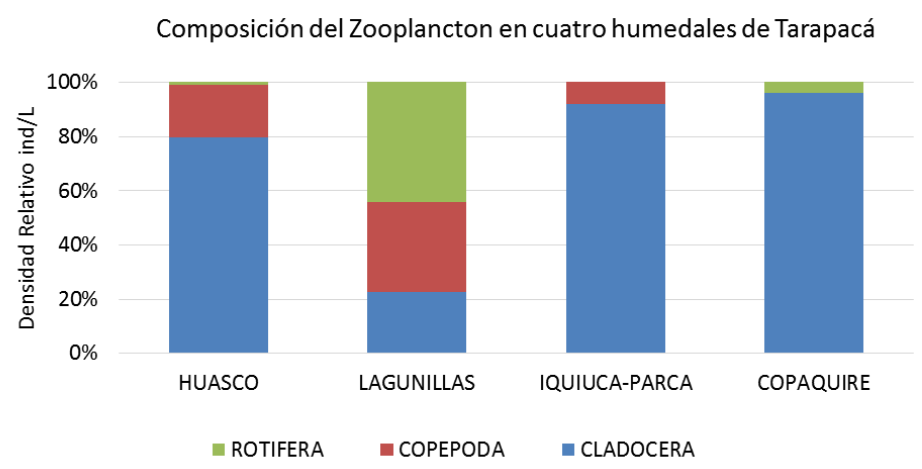

Figura 7. Composición taxonómica en el zooplancton de cuatro humedales de Tarapacá, observada de diciembre 2013 a mayo 2015, como densidad relativa (DR). 
Fluctuaciones de la densidad del fitoplancton y zooplancton: las densidades de fitoplancton en general presentaron una alta variabilidad temporal, haciéndose máximas durante la temporada de mayo 2014 en los cuatro humedales simultáneamente. Por otra parte, en la fracción zooplancton de quebrada Copaquire, se mantuvieron altas densidades durante las tres temporadas que fue monitoreado, con un máximo en diciembre 2014 y Huasco solo presentó altas densidades en mayo 2015 (figura 8).

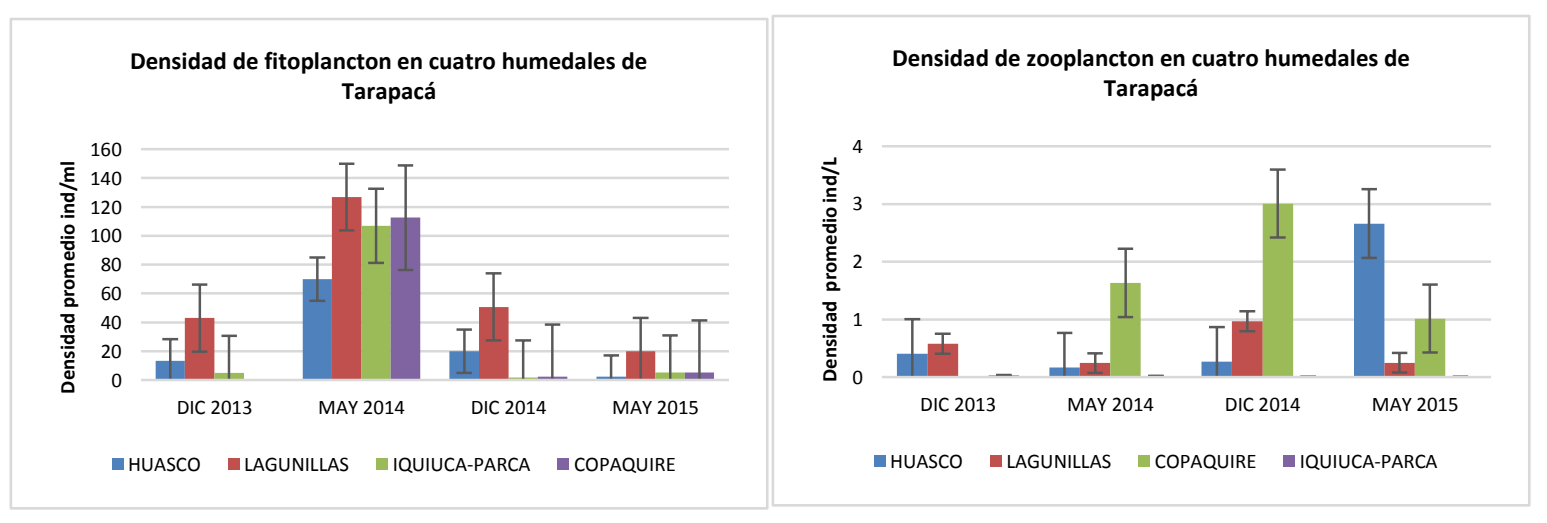

Figura 8. Densidades medias de fitoplancton y de zooplancton en cuatro humedales de Tarapacá durante cuatro temporadas climáticas

Tanto en lagunas como en quebradas, los patrones de fluctuación en la diversidad del fitoplancton son comparables entre humedales del mismo tipo, sobretodo en el caso de las quebradas. Laguna el Huasco, alcanzó niveles de diversidad mucho mayores que Lagunillas, con un máximo en la temporada de mayo 2014 (figura 9).
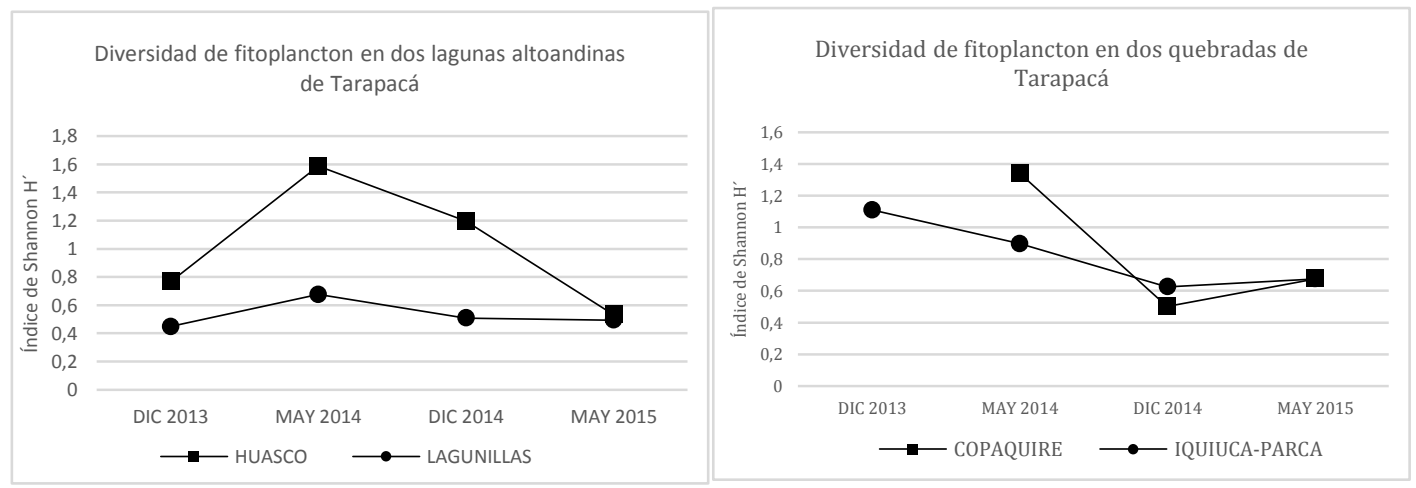

Figura 9. Fluctuaciones de diversidad de fitoplancton en lagunas y quebradas de Tarapacá.

En lagunas, el patrón de fluctuación de diversidad en zooplancton resultó ser similar en tres de las cuatro temporadas. Durante la temporada de mayo 2014, mientras Huasco presentó la una mínima diversidad de fitoplancton, Lagunillas por el contrario concentró una de las diversidades más altas del periodo. Y en las 
quebradas, las diversidades de zooplancton durante las tres temporadas comparables fluctuaron con un mismo patrón (figura 10).
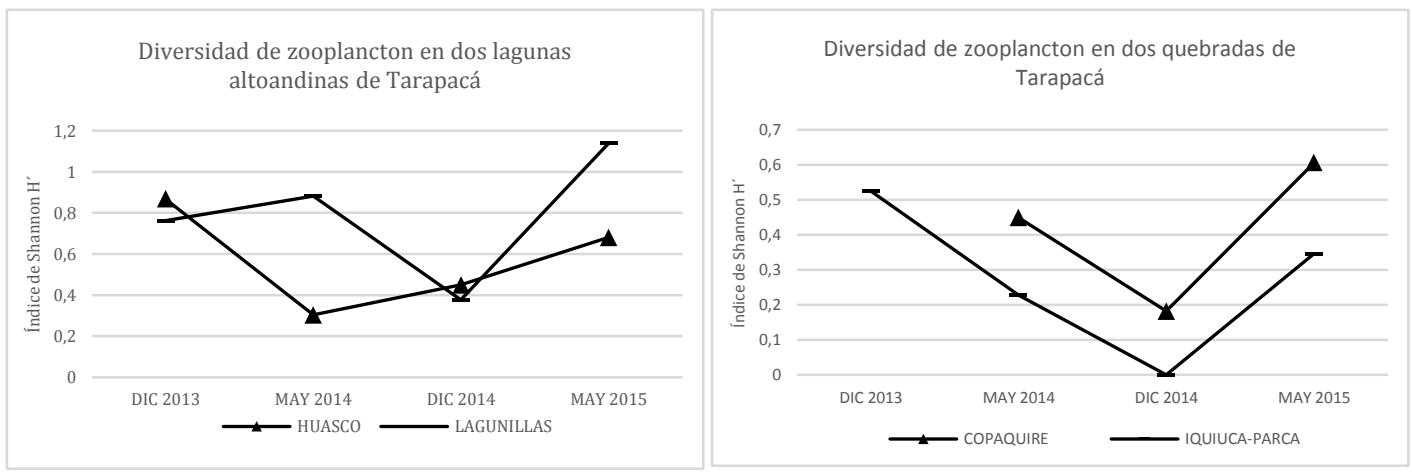

Figura 10. Fluctuaciones de diversidad de zooplancton en lagunas y en quebradas de Tarapacá.

Al promediar los valores de diversidad obtenidos en el fitoplancton de dos humedales similares y contrastarlos con los valores promediados de diversidad del zooplancton en los mismos, se aprecia que en lagunas (figura 11 A), las tendencias de variación en las diversidades de fito y zooplancton parecen invertirse cada tres temporadas climáticas, pero en quebradas, los patrones de variación en las diversidades son muy similares, disminuyendo y aumentando en proporciones semejantes (figura $11 \mathrm{~B}$ ).
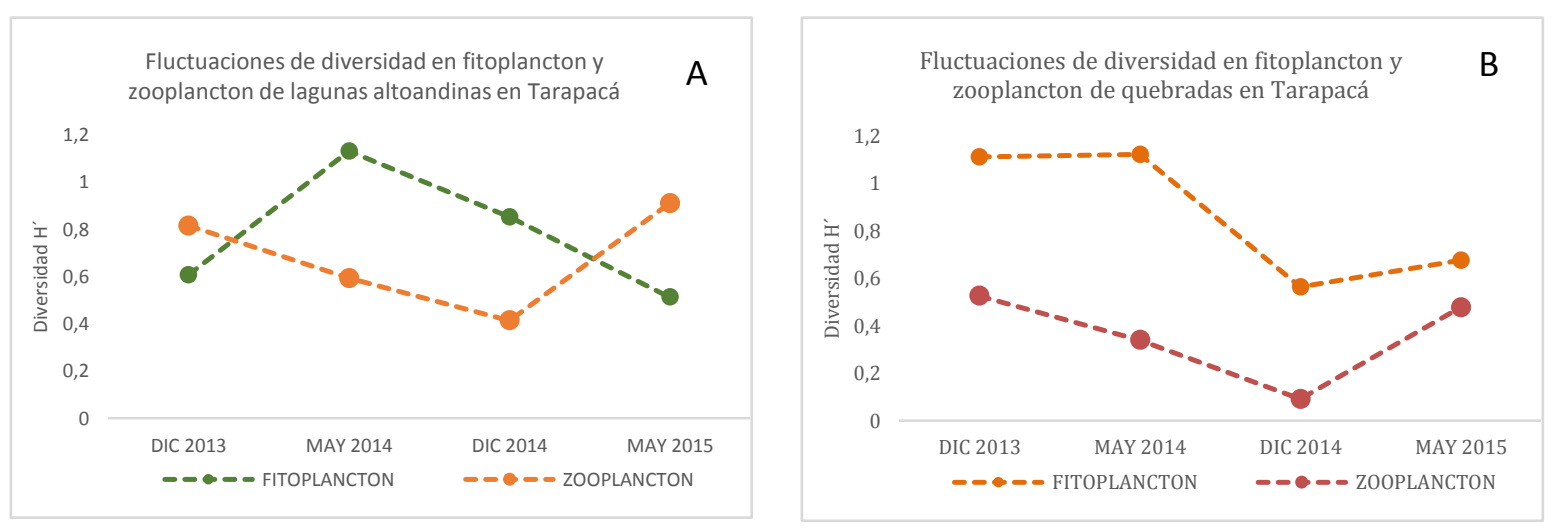

Figuras 11, A y B. Comparación de las fluctuaciones de diversidad de zooplancton y fitoplancton, en lagunas y en quebradas de Tarapacá.

Análisis de componentes principales entre parámetros fisicoquímicos y variables bióticas.-

Lagunas: los primeros dos ejes de componentes principales acumulan el 36,56\% de la varianza (figura 12). En lagunas, la densidad del zooplancton presentó fuerte correlación con los sólidos disueltos totales (Pearson $=0,66)$, la riqueza estuvo asociada positivamente con un $\mathrm{pH}$ más bien alcalino $($ Pearson= 0,41$)$ y 
Sustainability, Agri, Food and Environmental Research, 4(4), 2016: 27-44

ISSN: 0719-3726

temperaturas bajas (Pearson= $-0,38$ ), así mismo la diversidad $\mathrm{H}^{\prime}$ se relacionó inversamente con la temperatura $($ Pearson $=-0,50)$.

Por su parte, la diversidad de fitoplancton mantuvo una relación inversa con la riqueza de zooplancton $($ Pearson $=-0,35)$ y estuvo asociada a niveles bajos de $\mathrm{pH}($ Pearson $=-0,37)$.

Quebradas: los primeros dos ejes de componentes principales acumulan el 49,19\% de varianza (figura 13). En estos ecosistemas la densidad del zooplancton presentó una relación inversa con la temperatura $($ Pearson $=-0,55)$ y la riqueza presentó una relación inversa con los parámetros de temperatura $($ Pearson $=-0,87)$ y conductividad (Pearson= -0,57). Por otra parte, la diversidad de fitoplancton se relacionó inversamente con el $\mathrm{pH}($ Pearson $=-0,65)$ y la resistividad $($ Pearson $=-0,63)$.

Las estaciones de Iquiuca-Parca en general estuvieron asociadas a temperaturas medio-altas, baja oxigenación y altas densidades de fitoplancton, particularmente en las temporadas de diciembre 2014 y mayo 2015. Las estaciones de Copaquire se caracterizaron por ser altamente oxigenadas en diciembre 2014, con alta densidad, riqueza y diversidad de zooplancton en las temporadas de mayo.

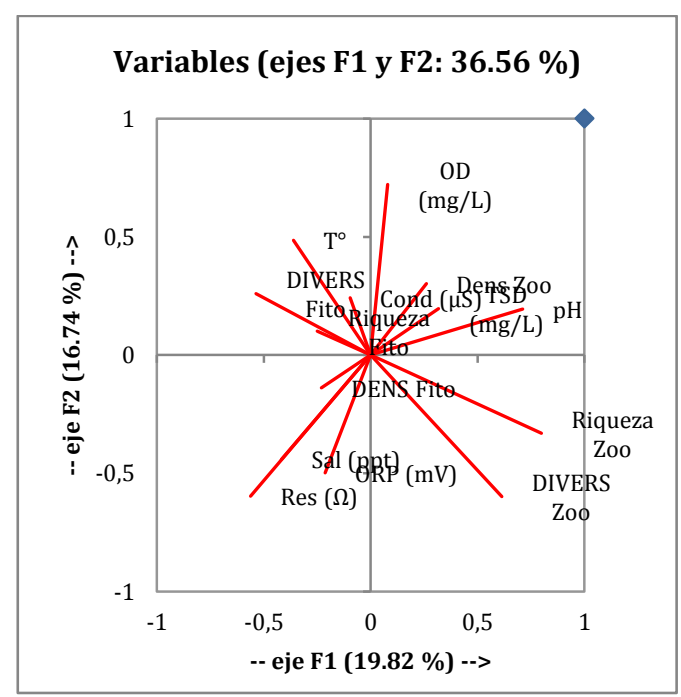

Figura 12. Proyección ortogonal de las dos primeras componentes entre variables bióticas y los parámetros fisicoquímicos en dos lagunas altoandinas de Tarapacá. 


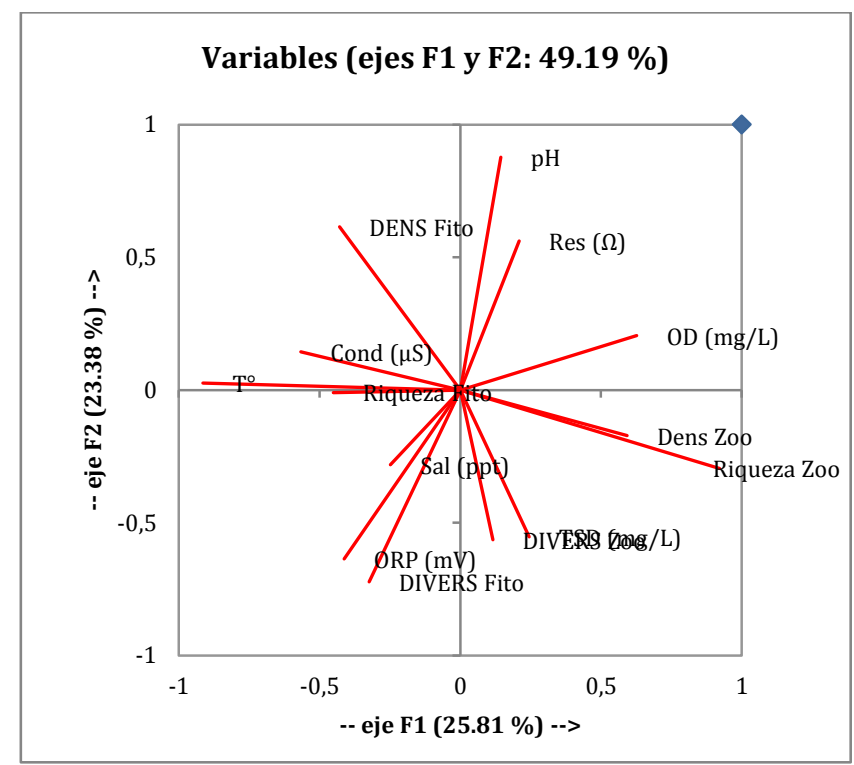

Figura 13. Proyección ortogonal de las dos primeras componentes entre variables bióticas y los parámetros fisicoquímicos en dos quebradas de Tarapacá.-

\section{Discusión}

Fitoplancton: en cuanto a la composición de los ensambles, a la cercanía taxonómica entre las comunidades de Huasco y Lagunillas debe sumarse el hecho de que los ensambles de ambos humedales están dominados numéricamente por dos diatomeas tales como Nitzschia Hassall, 1845 y Diatoma Bory de SaintVincent, 1824,. Esta predominancia por sobre otros clados, probablemente guarda relación con el hecho de que el frústulo otorga una ventaja frente cambios abruptos en los niveles del agua, insolación y el stress hídrico producto de la concentración de sales en el medio. Por su parte, las quebradas de Iquiuca-Parca y Copaquire comparten casi la mitad de los taxa del fitoplancton, sin embargo sus estructuras comunitarias son muy distintas.

Dorador et al. (2007) describen la presencia de Chroococcales en laguna el Huasco, como cianobacterias propias del sedimento y de poca presencia en la columna, por lo tanto su rareza a lo largo del periodo de estudio puede atribuirse razonablemente a su hábito bentónico y al método de muestreo utilizado en este trabajo.

Zooplancton: el género Attheyella Brady, 1880, es un taxón de amplia distribución geográfica (Hadel, 1986). Una especie de este género junto con sus copepoditos, están presentes en casi todas las estaciones y en altas densidades, conformando el 29\% de las densidad del zooplancton del salar del Huasco en mayo 2015. Por lo tanto, además de ser un taxón dominante, éste copépodo podría ser evaluado como una de las mayores fuentes de biomasa animal disponible para los flamencos. 
Sustainability, Agri, Food and Environmental Research, 4(4), 2016: 27-44

La presencia de Diaclyclops bisetosus (Rehberg, 1880) en las lagunas de Huasco, Lagunillas y un registro puntual en la quebrada de Copaquire durante el monitoreo de mayo 2014, agrega un nuevo reporte de presencia para una especie de distribución muy restringida en Chile, descrita solo para los cuerpos de agua dentro del Parque Nacional Lauca (Márquez-García et al, 2009), aumentando más al sur la distribución geográfica de esta especie en Chile.

En lagos y lagunas andinas, se ha descrito que los niveles de salinidad son un importante factor regulador de las estructuras comunitarias del zooplancton, determinando una baja riqueza de especies (De Los Ríos \& Crespo, 2004). En el caso de las lagunas de Huasco y Lagunillas, efectivamente se halló una relación inversa entre la salinidad y la riqueza de zooplancton (Pearson $=-0,15$ ) pero esta resultó sin significancia estadística, por lo tanto no fue posible confirmar el efecto. A pesar de esto, se encontraron especies clasificadas como halofílicas (el caso de B. popoensis), habitantes exclusivos de ambientes con salinidades de 1-9 ppt (Bayly, 1993; Williams et al., 1995; Menu-Marquet al., 2000) pero en Chile incluso se la ha reportado en ambientes con niveles hipersalinos de 45 g/L a 90 g/L (De Los Ríos \& Crespo, 2004).

Chidorus sphaericus (Müller, 1776) es un microcrustáceo propio de aguas dulces y sistemas lénticos, presente en la mayoría de los grandes lagos de Chile, desde el lago Chungará en el norte, hasta lago Llanquihue en el sur (De Los Ríos-Escalante, 2010). Fue detectado en laguna Huasco casi exclusivamente en vertientes, mientras que en Lagunillas estuvo presente tanto en las vertientes como en la laguna, cuando la salinidad no superó los 0,6 ppt. Los cladóceros han sido descritos como el grupo de microcrustáceos más abundante de los sistemas límnicos y por lo tanto de gran influencia en la regulación de las comunidades del zooplancton en estos (Martínez y Montecino, 2000). Particularmente Daphnia Müller, 1785 ha sido señalado en forma recurrente como un taxa con un consumo amplio de partículas alimentarias, lo cual en principio facilitaría su coexistencia con otras especies. A lo largo de éste estudio, solo se detectó Daphnia sp en una vertiente de Huasco, pero en una altísima densidad (240 ind/ml). Cabe señalar que estos microcrustáceos son organismos filtradores y detritívoros, que ejercen un rol importante en la depuración del agua y el ciclo del material orgánico suspendido, siendo afectados principalmente por la fluctuación en los niveles de agua, los niveles de insolación y los eventos de hipoxia (Locascio de Mitrovich et al 2005).

Aunque los rotíferos son en general organismos dominantes en aguas dulces, Brachinus plicatilis (Müller, 1786) es una especie halofílica, que puede habitar salinidades de 1 a $97 \mathrm{~g} / \mathrm{L}$ (Walker, 1981; Williams et al., 1995). Están presente en las vertientes y en espejo de Lagunilla, así como en Copaquire, en este caso parece presentar una baja tolerancia a la salinidad, ya que solo presentó abundancias importantes , particularmente en este último, donde alcanzó una densidad máxima en un medio de baja salinidad (0,63 ppt). El otro rotífero identificado fue una especie de Trichocerca Lamarck, 1801, en Huasco durante mayo 2015, en una salinidad 
Sustainability, Agri, Food and Environmental Research, 4(4), 2016: 27-44

ISSN: 0719-3726

máxima de 1,77 ppt. Se sabe que los rotíferos poseen la capacidad de alterar las dinámicas tróficas en las comunidades planctónicas, por lo tanto su rol como recicladores de nutrientes no debiera subestimarse (Derry et al., 2003).

A nivel genérico, Artemia predomina solo en ambientes de salinidad mayor a 90 ppt (Williams et al., 1995). Artemia franciscana Kellogg, 1906 fue identificada en laguna el Huasco, en densidades bajas y con salinidades de 9,54 ppt en mayo 2014 y 33,2 ppt en mayo 2015, muy por debajo del rango mínimo de 45 ppt reportado en Chile por De Los Ríos \& Salgado (2012) en lagunas de la zona central.

A diferencia de lo que describe De Los Ríos-Escalante (2010), para la generalidad de los ecosistemas de aguas interiores de Chile, en ambas lagunas se evidenció un marcado predominio de copépodos tanto Cyclopoideos (D. bisetosus) como Harpacticoideos (Atteyella $\mathrm{sp}$ ). Los ensambles de las quebradas estuvieron conformados casi exclusivamente por Cladóceros Chydoridae Stebbing, 1902, con representaciones menores de rotíferos (Brachionus plicatilis Müller, 1786) y copépodos (Attheyella sp).

Hulbert \& Chang (1983) en un estudio manipulativo, cercaron cuatro áreas dentro de una laguna altiplánica de Bolivia para impedir la alimentación de Phoenicoparrus andinus (Philippi, 1854) en ellas. Luego de tres semanas de exclusión observaron que diatomeas del género Surirella Turpin, 1828 cubrieron completamente el fondo de la laguna (bentos), mientras que en las superficies de la laguna, su cobertura era de aproximadamente el 50\%. También detectaron que hubo diferencias en la composición de especies de plancton entre las áreas comparadas. Esto demuestra que el forrajeo por flamencos tiene una fuerte influencia en la conformación de los ensambles y particularmente en la densidad de las diatomeas más abundantes.

Ambas lagunas son sitios de alimentación para flamencos durante todo el año, por lo tanto ambos ensambles se encuentran sometidos a presión de depredación. El rol de los flamencos y otras aves acuáticas filtradores, no ha sido observado en este estudio, pero es un factor que debe considerarse al momento de interpretar los resultados. Se sabe que la distribución de $P$. andinus se correlaciona estrechamente con la presencia de diatomeas del género Surirella, las cuales a su vez suelen ser dominantes en lagunas del altiplano (Hulbert \& Chang, 1983).

Cabe mencionar que existen poblaciones de Orestias Agassi Valenciennes, 1846, una especie endémica y en peligro de extinción que habita las vertientes de laguna el Huasco, respecto de la cual se sabe que posee una dieta estenofágica compuesta al menos en un $50 \%$ por organismos zooplanctónicos, con alta selectividad sobre cladóceros y ostrácodos (Guzmán \& Sielfeld 2009; Riveros et al., 2012; Hurlbert et al., 1986). Son depredadores importantes de zooplancton e invertebrados acuáticos capaces de establecer fuerte competencia con los flamencos, donde una alta presencia de peces sería un predictor de baja abundancia de estas aves (Hurlbert et al., 1986). Una relación que ha sido descrita resumidamente por Jaksic (1997) como un ejemplo de competencia 
interespecífica asimétrica entre taxa distantes, en que los peces afectan el patrón de distribución del flamenco chileno, pero no a la inversa. Dados estos antecedentes, es esperable que cambios importantes en las densidades y la diversidad del ensamble zooplanctónico en las vertientes, tengan impactos directos en la disponibilidad de alimento para peces y aves filtradoras, una relación que quizás amerite más atención en futuros estudios, que incluso podrían extenderse a Trichomycterus rivulatus Valenciennes, 1846.

Fluctuaciones y parámetros: el Salar del Huasco, tanto en sus vertientes como en laguna, presentó alta riqueza y diversidad de fitoplancton de manera constante a lo largo del año, sin diferencias marcadas entre las temporadas de mayo y diciembre. Por su parte, laguna Lagunillas presentó alta riqueza y diversidad de zooplancton en sus vertientes y altas densidades de fitoplancton durante las temporadas de mayo, pero en diciembre tanto la laguna como las vertientes se caracterizaron por mayores densidades de zooplancton.

Las tendencias de fluctuación de diversidad en lagunas tienen una ciclicidad que cambia cada tres temporadas y donde los patrones de fluctuación en la diversidad del fitoplancton y en el zooplancton parecen ser inversos. Por su parte los ciclos de variación de la diversidad de los ensambles en las quebradas también cambian cada tres temporadas, pero en este caso las tendencias de cambio son las mismas.

Finalmente, queda claro que los ensambles planctónicos en las lagunas fueron afectados mayormente por parámetros como la temperatura, el oxígeno disuelto y el pH, relacionados con la ventilación, mientras que en las quebradas los ensambles fueron afectados primordialmente por parámetros como la salinidad y otros sólidos disueltos, más relacionados con condiciones de suelo y cambios a lo largo de la escorrentía.

\section{Agradecimientos}

Financiamiento y soporte para este estudio por parte de Centro de Estudios del Desarrollo-Centro de Estudios de Humedales en el marco del proyecto institucional "Observatorio de los efectos del cambio climático sobre la biodiversidad de los humedales de Tarapacá”. A David Uribe por su ayuda con procedimiento en software R.

\section{Bibliografía}

Araya, J., Zuñiga, L. 1985. Manual taxonómico del zooplancton lacustre de Chile. Boletín Informativo Limnológico. Valdivia Chile. Pág. 110.

Arocena, R. Conde, D. 1999. Métodos en ecología de aguas continentales con ejemplos de Limnología en Uruguay. DI.R.A.C. - Facultad de Ciencias. Universidad de la República, Uruguay 233 pp. 
Sustainability, Agri, Food and Environmental Research, 4(4), 2016: 27-44

Bicudo, C.E., Menezes M. 2005. Gêneros de algas de águas continentais do Brasil: chave para identificação e descrições. 1 ed. São Carlos: RiMa Editora. 489 pp.

Chapman, M.A., Burns C.W. 1976. Sexual and other differences in copepodite stages of some New Zealand Calamoecia and Boeckella spp. (Copepoda: Calanoida). New Zealand Journal of marine and Freshwater research 10 (1) 131-137.

De los Ríos, P., Crespo J.E., 2004. Salinity effects on the abundance of Boeckella poopoensis (Copepoda, Calanoida) in saline ponds in the Atacama Desert, northern Chile. Crustaceana, 77: 417-423.

De los Ríos, P., Salgado, I. 2012. Artemia (Crustacea, Anostraca) in Chile: a review of basic and applied biology. Latin American Journal of Aquatic research 40: 487-497.

De los Ríos-Escalante, P. 2010. Crustacean Zooplankton Communities in Chilean Inland Waters. Crustaceana Monographs, 12. Koninklijke Brill NV, Leiden.

De los Ríos-Escalante, P., Meruane, J., Morales, M.C., Rudolph, E., Fuentealba. C., Boxshall G. 2013. Zoogeography of Chilean inland water crustaceans. Latin american journal of aquatic research, 41: 846-853.

Derry, A.M., Hebert P.D.N., Prepas, E.E. 2003. Evolution of rotifers in saline and subsaline lakes: A molecular phylogenetic approach. Limnology \& Oceanography, 48: 675-685.

Dorador, C., Pardo, R. Vila, I. 2003. Variaciones temporales de parámetros físicos, químicos y biológicos de un lago de altura: el caso del lago Chungará. Revista Chilena de Historia natural, 76: 15-22.

Guzmán, J.A., Sielfeld W., 2009. Dieta de Orestias agassii (cuvier \& valenciennes, 1846) (Teleostei: Cyprinidontidae) del salar del Huasco, norte de Chile. Gayana, 73: 17-27.

Hadel, V.F., 1986. Two new species of Attheyella (Copepoda: Harcpactoidea: Canthocapmtidae) from Bromeliads of the Serra Da Jureia (Sao Paulo, Brasil). Journal of Crustacean Biology 6: 777-778.

Hurlbert, S.H., Loayza, W., Moreno, T. 1986. Fish-flamingo-plankton interactions in the Peruvian Andes. Limnology \& Oceanography, 31: 457-468.

Jaksic, F. 1997. Ecología de los vertebrados de Chile. Primera edición. Ediciones Universidad Católica de Chile, Santiago, Chile. 262 pp.

Locascio de Mitrovich, C., Villagra De Gamundi, A., Juárez, J., Ceraolo, M. 2005. Características limnológicas y zooplancton de cinco lagunas de la Puna-Argentina. Ecología en Bolivia, 40: 10-24. 
Sustainability, Agri, Food and Environmental Research, 4(4), 2016: 27-44

ISSN: 0719-3726

Marquez-García, M., Vila, I., Hinojosa, L., Mendez, M., Carvajal, J.L., Sabando, M. 2009. Distribution and seasonal fluctuations in the aquatic biodiversity of the southern Altiplano. Limnologica 39: 314-318.

Martinez, G., Montecino, V. 2000. Competencia en Cladocera: implicancias de la sobreposición en el uso de los recursos tróficos. Revista Chilena de Historia Natural, 73: 787-795.

McAleece, N. 1997. BioDiversity professional beta 1.Version 1.0. The Natural History Museum and The Scottish Association for Marine Science.

Disponible en http://www.nhm.ac.uk/zoology.bdpro.

Niemeyer, H. , Cereceda, P., 1984. Geografía de Chile: Hidrografía. Instituto Geográfico Militar.

Parra, O., Bicudo, C.M., 1996. Algas de Aguas Continentales: Introducción a la Biología y Sistemática. Ediciones Universidad de Concepción. 268 pp.

Peña, H., Grilli, A., Salazar, C., Orphanopoulos, D., Suzuki, O., Aravena, R., Rauert A., (1989) Isotope hydrology investigation, area of the Salar de Llamara, Atacama Desert, Chile. (IAEA-TECDOC--502). International Atomic Energy Agency (IAEA)

R Core Team (2015) R: A language and environment for statistical computing. -R Foundation for Statistical Computing. Disponible en www.r-project.org

Risacher, F., Alonso „H., Salazar, C. 1999. Geoquímica de aguas en cuencas cerradas: I, II y III regiones, Chile: 221-230. (Technical Report of the Dirección General de Aguas, Chile, Universidad Católica del Norte, Chile, and the Institut de Recherche pour le Développement, France).

Riveros, J., Vila, I., Méndez, M., 2012. Nicho trófico de Orestias agassii (Cuvier \& Valenciennes, 1846) del

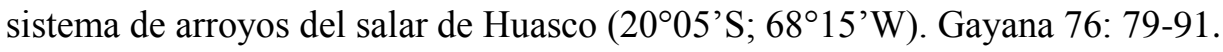

Sant'Anna, C., Azevedo, M.T., Agujaro, L.F., De Carvalho, L.R.M., Souza R.C.R. 2006. Manual ilustrado para identificação e contagem de cianobacterias planctônicas de águas continentais brasileiras. Rio de Janeiro: Interciência. $58 \mathrm{pp}$.

Utermöhl, H. 1958. Zur Vervollkommnung der quantitativen Phytoplankton-Methodik. Mitteilingen International Vereinung fur Theoretishe und Angewaldte Limnolologie, 9:1-38.

Villalobos, L. 2006. Estado del conocimiento de los crustáceos zooplanctonicos dulceacuicolas de Chile. Gayana 70: 31-39. 
Sustainability, Agri, Food and Environmental Research, 4(4), 2016: 27-44

Walker, K.F. 1981. Synopsis of ecological information on the saline lake rotifer Brachionus plicatilis Mfuller 1786. Hydrobiologia 81: 159-167.

Willamson, C.E., Reid, J.K. 2001. Copépoda. En: Ecology and Classification of North American Freshwater Invertebrates, Thorp J.H. \& Covich A.P. Editores, $2^{\circ}$ ed. Pág: 915-951. Academic Press. San Diego, California. Estados Unidos.

Williams, W. D., Carrick, T. R., Bayly, I. A., Green, J., Herbst, D. B. 1995. Invertebrates in salt lakes of the Bolivian Altiplano. International Journal of Salt Lake Research, 4: 65-77. 